\title{
SEPSIS SCREEN PARAMETERS AS A TOOL FOR DIAGNOSIS OF NEONATAL SEPSIS
}

\author{
Zeba Shamshad Ali1, Bharat Ankush Ghodke2, Aradhana Bhubaneswar Deka ${ }^{3}$ \\ ${ }^{1}$ Assistant Professor, Department of Pathology, Grant Medical College \& Sir JJ Hospital, Mumbai. \\ ${ }^{2}$ Assistant Professor, Department of Pathology, Grant Medical College \& Sir JJ Hospital, Mumbai. \\ ${ }^{3}$ Blood Transfusion Officer, Grant Medical College \& Sir JJ Hospital, Mumbai.
}

\section{ABSTRACT}

\section{BACKGROUND}

Infections are a frequent and important cause of morbidity and mortality in the neonatal period. Neonatal sepsis is one of the leading causes of morbidity and mortality in India. It is probably responsible for $30-50 \%$ of the total neonatal deaths each year in developing countries. Early recognition, diagnosis and treatment of serious infection in neonates are essential because of the risk of permanent morbidity and mortality.

Aims and Objectives of the study are to evaluate various haematological parameters singly, find out sensitivity and specificity of the various sepsis screen parameters in clinically suspected cases of neonatal septicaemia and to correlate them with blood culture.

\section{MATERIALS AND METHODS}

The present study is an observational study of the haematologic profiles (screening parameters) of 130 neonates admitted in the neonatal care unit of our tertiary care hospital from January 2013 to June 2014. All neonates asymptomatic and symptomatic were subjected to sepsis screen parameters after taking the clinical history and all investigations were done before starting any antibiotics.

\section{RESULTS}

Out of total 130 cases, 60 (46.1\%) were culture positive and 70 (53.9\%) were culture negative. Amongst 60 culture positive cases, $38(63.4 \%)$ were EOS whereas $22(36.6 \%)$ were LOS. Amongst 70 culture negative cases, $24(34.3 \%)$ were EOS whereas 46 $(65.7 \%)$ were LOS. Overall there is male preponderance. Preterm babies outnumbered the term babies.

\section{CONCLUSION}

Early onset sepsis (early neonatal period) was more common than late onset sepsis. Gram-negative infection was more common, Klebsiella was the most common organism isolated. C-reactive protein had highest sensitivity among all. Micro-ESR had highest specificity. All the tests employed using haematological profile can be performed easily at the primary health care centre level and results can be obtained in an hour.

\section{KEYWORDS}

Sepsis, ESR, Culture.

HOW TO CITE THIS ARTICLE: Ali ZS, Ghodke BA, Deka AB. Sepsis screen parameters as a tool for diagnosis of neonatal sepsis. J. Evolution Med. Dent. Sci. 2017;6(79):5584-5588, DOI: 10.14260/jemds/2017/1212

\section{BACKGROUND}

Infections are a frequent and important cause of morbidity and mortality in the neonatal period. Neonatal sepsis is one of the leading causes of morbidity and mortality in India. It is probably responsible for $30-50 \%$ of the total neonatal deaths each year in developing countries. Neonatal sepsis is a clinical syndrome of bacteraemia characterised by systemic signs and symptoms of infection in the first month of life. ${ }^{1}$

Probable sepsis is defined as presence of clinical signs of bacterial infection associated with positive laboratory tests in the absence of blood culture positivity.

The worldwide incidence of sepsis is 1500 cases per million (1999). Angus et al reported 750000 cases per year (3 in 1000 persons) in the United States. The average mortality of $30 \%$, equivalent to 250000 deaths per year, can increase to

'Financial or Other Competing Interest': None.

Submission 11-09-2017, Peer Review 24-09-2017,

Acceptance 27-09-2017, Published 30-09-2017.

Corresponding Author:

Dr. Aradhana Bhubaneswar Deka,

Regional Blood Bank, St. George's Hospital,

Mumbai - 400001.

E-mail: aradhana.deka@gmail.com

DOI: $10.14260 /$ jemds/2017/1212

\section{(c) (1) (8) $\Theta$}

$60 \%$ or more when multiple failures occur in more than three organs for 3 or more days. $(9,20,21)$. The Atlanta Centers for Disease Control report negative coagulase Staphylococcus, Staphylococcus aureus, Enterococcus and Candida as causes of sepsis. ${ }^{2}$ Incidence in full term babies is $1 / 1000$ live births, in pre-term is 4 cases/1000 births and in very low birth weight babies is 300 cases/1000.

\section{Aims and Objectives of the Study}

To evaluate various haematological parameters singly, find out sensitivity and specificity of the various sepsis screen parameters in clinically suspected cases of neonatal septicaemia and to correlate them with blood culture.

\section{MATERIALS AND METHODS}

The present study is an observational study of the haematologic profiles (screening parameters) of 130 neonates admitted in the neonatal care unit of our tertiary care hospital from January 2013 to June 2014.

\section{Inclusion Criteria}

All asymptomatic cases born to a mother with risk factor, symptomatic cases born to a mother with or without risk 
factor with age up to 28 days and birth weight more than 500 grams were included in our study.

\section{Exclusion Criteria}

All cases with age more than 28 days, birth weight $<500$ grams, neonates with congenital anomalies and neonates who were given antibiotics before collection of sample were excluded from our study. Data collected as per maternal history of fever $>72$ hours prior to delivery, rash, leucorrhoea, premature rupture of membrane, prolonged labour, neonatal history of age and birth weight with signs and symptoms of infection, feeding difficulty, respiratory distress, irritability, oliguria, jaundice, diarrhoea, convulsion, hypothermia. All neonates asymptomatic and symptomatic were subjected to sepsis screen parameters after taking the clinical history and all investigations were done before starting any antibiotics. Sepsis screen parameters included in the present study were-

1. Total white cell count.

2. Absolute neutrophil count.

3. Immature to total neutrophil count ratio.

4. Platelet count.

5. Micro- ESR.

6. C-reactive protein.

Individual parameters were compared with blood culture report.

Under all aseptic precautions, cleaning the surface with Betadine spirit with gloves in hand, blood samples were collected by venepuncture. The $0.5 \mathrm{cc}$ of blood of neonate was collected in vacutainer tube with trisodium EDTA as an anticoagulant and culture tube. Total leucocyte count, platelet count and absolute neutrophil count was obtained from automated electronic cell counter. Peripheral smear was prepared with one drop of blood and then stained with Leishman stain, mounted with mounting medium (DPX) with a clean and dry $25 \times 25 \mathrm{~mm}$ coverslip. Stained peripheral smears were examined under oil immersion, differential count and band forms were counted. Band counts were estimated from first 100 neutrophils in the smear. Band forms were defined as neutrophils with lobes connected by a band or neutrophil which had nuclei without lobulations.

$\mathrm{I}: \mathrm{T}$ ratio $=$ Band $/$ Total neutrophil per $100 \mathrm{WBCs}$ X 100

C-Reactive Protein- $1 \mathrm{cc}$ blood was collected in plain bulb. CRP was estimated by latex agglutination method with serum based kit and test was done as per manufacturer's instruction. In the kit a special black colour card was provided, with 6 wells. The undiluted patient's serum was added and mixed with CRP reagent in one well, with positive and negative controls set up in the other two. Agglutination was appreciated after two minutes if the test serum was positive and further diluted to find the titres. Normal CRP was less than $1 \mathrm{mg} / \mathrm{dL}$.

Positive above $1 \mathrm{mg} / \mathrm{dL}$ in titres of 1:12, 1:24 and so on.

Micro-ESR- small amount of anti-coagulant blood was collected in capillary tube $(75 \times 1.1 \mathrm{~mm})$ for estimation of $\mathrm{m}$ ESR. Care is taken to prevent air entry in the tube. Lower end of tube was fixed vertically on stand. The distance from top of tube to meniscus of packed WBC was recorded in $\mathrm{mm}$ at the end of 1 hour.

An estimate of normal reading is: any value $>$ age (days) + 3 up to maximum of $15 \mathrm{~mm} / \mathrm{hr}$.
Blood culture- All samples (1-2 mL) were sent to the Department of Microbiology in the conventional Hartley's blood culture broth and Automated BacT/Alert culture system and the reports were obtained after $72 \mathrm{hrs}$.

Cultures were reported as negative when they did not yield any growth at the end of 7 days.

For diagnosing neonatal sepsis, we need a useful screening protocol where a balance must be achieved between sensitivity and specificity. Sensitivity is an expression of the probability that the test is positive in the presence of sepsis and specificity is an expression of the probability that same test will be negative in absence of the sepsis. ${ }^{3}$ The statistical calculations were done online in the following website: medcalc.org.

\section{RESULTS}

Present study is comprised of 130 cases with clinical suspicion of neonatal sepsis. All cases were studied as per the proforma. Cases were subjected for sepsis screen tests and observations were compared with blood culture results. The observations in the present study were as follows:

Out of total 130 cases, $60(46.1 \%)$ were culture positive and $70(53.9 \%)$ were culture negative. Table 2 shows total 130 cases, out of which $62(47.7 \%)$ were EOS whereas 68 (52.3\%) were LOS. Amongst 60 culture positive cases, 38 (63.4\%) were EOS whereas 22 (36.6\%) were LOS. Amongst 70 culture negative cases, $24(34.3 \%)$ were EOS whereas 46 $(65.7 \%)$ were LOS. Overall there is male preponderance. Among the culture positive cases, preterm babies outnumbered the term babies. 70 culture negative cases, 19 (27.1\%) had birth weight $<2.5 \mathrm{~kg}$ whereas 51 (72.9\%) had birth weight $>2.5 \mathrm{~kg}$. In culture positive cases, low birth weight babies were more.

The study group was assessed for history of maternal risk factors. It was found that $48 \%$ cases had history of premature rupture of membrane, $15 \%$ cases had history of Urinary tract infection, $12 \%$ cases had history of fever and $34 \%$ cases had history of prolonged labour.

Culture positive and culture negative cases were compared based on history of maternal risk factors.

Premature rupture of membrane: Culture positive group had higher percentage of cases (53\%) than the $43 \%$ of cases in culture negative group. But this difference in frequency of positive history of PROM in the two groups was not found to be statistically significant.

Urinary tract infection: Culture positive group had higher percentage of cases $(23 \%)$ than the $11 \%$ of cases in culture negative group and this was statistically significant.

Fever: Culture positive group had higher percentage of cases $(20 \%)$ than the $6 \%$ of cases in culture negative group and this was statistically significant.

Prolonged labour: Culture positive group had low percentage of cases $(13 \%)$ than the $51 \%$ of cases in culture negative group and this.

\begin{tabular}{|c|c|c|}
\hline Blood Culture & Frequency & Percent \\
\hline Positive & 60 & 46.1 \\
\hline Negative & 70 & 53.9 \\
\hline Total & $\mathbf{1 3 0}$ & $\mathbf{1 0 0}$ \\
\hline Table 1. Proven Sepsis (Blood Culture Positive) (n=130) \\
\hline
\end{tabular}

n- Total number of cases. 


\begin{tabular}{|c|c|c|c|c|c|c|}
\hline \multirow{2}{*}{ Age } & \multicolumn{2}{|c|}{$\begin{array}{c}\text { Culture Positive } \\
\text { (n1=60) }\end{array}$} & \multicolumn{2}{c|}{$\begin{array}{c}\text { Culture Negative } \\
\text { (n2=70) }\end{array}$} & \multicolumn{2}{|c|}{ Total } \\
\cline { 2 - 7 } & Frequency & $\mathbf{\%}$ & Frequency & $\mathbf{\%}$ & Frequency & $\mathbf{\%}$ \\
\hline $\begin{array}{c}\text { EOS } \\
(0-3 D)\end{array}$ & 38 & 63.4 & 24 & 34.3 & 62 & 47.7 \\
\hline $\begin{array}{c}\text { LOS } \\
(4-28 D)\end{array}$ & 22 & 36.6 & 46 & 65.7 & 68 & 52.3 \\
\hline Total & $\mathbf{6 0}$ & $\mathbf{1 0 0}$ & $\mathbf{7 0}$ & $\mathbf{1 0 0}$ & $\mathbf{1 3 0}$ & $\mathbf{1 0 0}$ \\
\hline
\end{tabular}

Table 2. Age wise Distribution of Cases $(n=130)$

n-total number of cases, n1-total number of culture positive cases, n2-total number of culture negative cases, EOS- Early onset sepsis, LOS-Late onset sepsis, D- Days.

\begin{tabular}{|c|c|c|c|c|c|c|}
\hline \multirow{2}{*}{ Sex } & \multicolumn{2}{|c|}{$\begin{array}{c}\text { Culture Positive } \\
\text { (n1=60) }\end{array}$} & \multicolumn{2}{c|}{$\begin{array}{c}\text { Culture Negative } \\
\text { (n2=70) }\end{array}$} & \multicolumn{2}{c|}{ Total } \\
\cline { 2 - 7 } & Frequency & $\mathbf{\%}$ & Frequency & $\mathbf{\%}$ & Frequency & $\mathbf{\%}$ \\
\hline Male & 34 & 56.6 & 38 & 54.3 & 72 & 55.4 \\
\hline Female & 26 & 43.4 & 32 & 45.7 & 58 & 44.6 \\
\hline Total & $\mathbf{6 0}$ & $\mathbf{1 0 0}$ & $\mathbf{7 0}$ & $\mathbf{1 0 0}$ & $\mathbf{1 3 0}$ & $\mathbf{1 0 0}$ \\
\hline
\end{tabular}

Table 3. Sex wise Distribution of Cases $(n=130)$

n- Total number of cases, n1-total number of culture positive cases, $\mathrm{n} 2$-total number of culture negative cases.

\begin{tabular}{|c|c|c|c|c|c|c|}
\hline \multirow{2}{*}{$\begin{array}{c}\text { Gestational } \\
\text { Age }\end{array}$} & $\begin{array}{c}\text { Culture Positive } \\
\text { (n1=60) }\end{array}$ & $\begin{array}{c}\text { Culture Negative } \\
\text { (n2=70) }\end{array}$ & \multicolumn{2}{|c|}{ Total } \\
\cline { 2 - 7 } & Frequency & \% & Frequency & \% & Frequency & \% \\
\hline Preterm & 42 & 70 & 34 & 48.6 & 76 & 58.5 \\
\hline Term & 18 & 30 & 36 & 51.4 & 54 & 41.5 \\
\hline Total & $\mathbf{6 0}$ & $\mathbf{1 0 0}$ & $\mathbf{7 0}$ & $\mathbf{1 0 0}$ & $\mathbf{1 3 0}$ & $\mathbf{1 0 0}$ \\
\hline
\end{tabular}

Table 4. Gestational Age wise Distribution of Cases (n=130)

n- Total number of cases, n1-total number of culture positive cases, and n2-total number of culture negative cases.

\begin{tabular}{|c|c|c|c|c|c|c|}
\hline \multirow{2}{*}{$\begin{array}{c}\text { Birth } \\
\text { weight }\end{array}$} & \multicolumn{2}{|c|}{$\begin{array}{c}\text { Culture } \\
\text { Positive(n1=60) }\end{array}$} & \multicolumn{2}{c|}{$\begin{array}{c}\text { Culture } \\
\text { Negative(n2=70) }\end{array}$} & \multicolumn{2}{c|}{ Total } \\
\cline { 2 - 7 } & Frequency & $\mathbf{\%}$ & Frequency & $\mathbf{\%}$ & Frequency & $\mathbf{\%}$ \\
\hline$<2.5 \mathrm{~kg}$ & 37 & 61.7 & 19 & 27.1 & 56 & 43.0 \\
\hline$>2.5 \mathrm{~kg}$ & 23 & 38.3 & 51 & 72.9 & 74 & 57.0 \\
\hline Total & $\mathbf{6 0}$ & $\mathbf{1 0 0}$ & $\mathbf{7 0}$ & $\mathbf{1 0 0}$ & $\mathbf{1 3 0}$ & $\mathbf{1 0 0}$ \\
\hline
\end{tabular}

Table 5. Birth Weight wise Distribution of Cases $(n=130)$

n- Total number of cases, n1-total number of culture positive cases, $\mathrm{n} 2$-total number of culture negative cases.

\begin{tabular}{|c|c|c|c|c|c|c|c|c|}
\hline \multirow{2}{*}{\multicolumn{2}{|c|}{ Maternal Risk Factors }} & \multicolumn{2}{|c|}{ Culture Positive (n1=60) } & \multicolumn{2}{|c|}{ Culture Negative (n2=70) } & \multicolumn{2}{|c|}{ Total $(n=130)$} & \multirow[t]{2}{*}{ p Value } \\
\hline & & Frequency & $\%$ & Frequency & $\%$ & Frequency & $\%$ & \\
\hline \multirow{2}{*}{$\begin{array}{l}\text { PROM- Premature } \\
\text { rupture of membrane }\end{array}$} & Yes & 32 & 53.3 & 30 & 42.9 & 62 & 47.7 & \multirow{2}{*}{0.233} \\
\hline & No & 28 & 46.7 & 40 & 57.1 & 68 & 52.3 & \\
\hline \multirow{2}{*}{$\begin{array}{l}\text { UTI-Urinary tract } \\
\text { infection }\end{array}$} & Yes & 14 & 23.3 & 8 & 11.4 & 20 & 15.4 & \multirow{2}{*}{$0.020^{*}$} \\
\hline & No & 46 & 76.7 & 62 & 88.6 & 110 & 84.6 & \\
\hline \multirow{2}{*}{ Fever } & Yes & 12 & 20 & 4 & 5.7 & 16 & 12.3 & \multirow{2}{*}{$0.013^{*}$} \\
\hline & No & 48 & 80 & 66 & 94.3 & 114 & 87.7 & \\
\hline \multirow{2}{*}{ Prolonged labour } & Yes & 8 & 13.3 & 36 & 51.4 & 44 & 33.8 & \multirow{2}{*}{$<0.01^{*}$} \\
\hline & No & 52 & 86.7 & 34 & 48.6 & 86 & 66.2 & \\
\hline
\end{tabular}

$\mathrm{N}$-total number of cases, n1-total number of culture positive cases, n2-total number of culture negative cases, *statistically significant.

Total cases do not match because of overlapping of symptoms.

\section{DISCUSSION}

Neonatal sepsis is a serious illness with high morbidity as well as mortality. Though it is a life threatening condition, it is treatable due to advancement in antibiotic therapy. Therefore, diagnostic tests with greater sensitivity are desirable. Although blood culture is the most definitive test for the diagnosis of neonatal sepsis, it has low sensitivity and leads to delay in the diagnosis for at least 48 hours. Therefore, in practice, paediatricians prescribe antibiotics empirically to avoid rapidity of clinical deterioration in septic newborn infants. Nowadays, prophylactic use of antibiotics came under vigorous scrutiny due to development of drug resistance, cost of unnecessary therapy and problems of drug toxicity. Thus, realising the importance of early and correct diagnosis of neonatal septicaemia, many workers have made an attempt to diagnose septicaemia by means of simple tests collectively called as "sepsis screen".

In present study, among culture positive cases, $63.4 \%$ of cases were in the age group of 1-3 days (early neonatal period) that is early onset sepsis was more common, may be because of maternal factors. Neonates may acquire the pathogens presumably transmitted perinatally from the mother. The findings of the present study correlates with Gupta et al, ${ }^{4}$ Nandy et al, ${ }^{5}$ Sriram et al. ${ }^{6}$

Wilson et al (1974) observed male predominance because congenital anomalies of urinary tract are attributable to higher prevalence of congenital anomalies in males and hence risk of urinary tract infection. ${ }^{7}$ In the present study, $56.6 \%$ culture positive cases were males and $43.4 \%$ were females with male: female ratio being 1.3:1.

The rate of infection is inversely proportional to the birth weight. ${ }^{8}$ Higher incidence of sepsis in low birth weight neonates is attributable to low maternal acquired IgG and inherent susceptibility to infection. ${ }^{9}$

In the present study, $61.7 \%$ of culture positive cases were low birth weight neonates. Present study is more comparable to Gupta et al study than other studies like Varsha et al,10 Swarnkar et al. ${ }^{11}$

The present study showed higher percentage of culture positivity among preterm neonates compared to term neonates. We found that $70 \%$ of culture positive cases were preterm neonates. The findings of the present study correlate with Shirazi et $\mathrm{al}^{12}$ and Basu et al. ${ }^{13}$ studies.

There is limitation in diagnosis of neonatal sepsis as there is no single test which meets criteria for ideal diagnostic test. There are four characteristics of laboratory tests one needs to consider when evaluating an infant for possible sepsis: sensitivity, specificity, positive predictive value and negative 
predictive value. A sensitive test will rarely miss an infant with sepsis and a specific test will rarely misclassify an infant who is healthy. Sensitivity is a desirable characteristic when the condition is serious and treatable, which is certainly true of infants with sepsis. Given that the treatment of neonatal sepsis has a relatively low toxicity, of the two, sensitivity is clearly more important. Positive predictive value and negative predictive value tell clinicians how to interpret a laboratory test. Positive predictive value is the probability that an infant is infected when the laboratory test is abnormal, while negative predictive value is the probability that an infant is healthy when the test is normal. If the goal is to only treat infants with high probability of infection, then clinicians must use screening tests with a high negative predictive value. ${ }^{14}$

In the present study, amongst all individual diagnostic tests, C-reactive protein had the highest sensitivity and negative predictive value. Micro-ESR had the highest specificity, thrombocytopenia had highest positive predictive value.

As per the individual tests are concerned, all the already mentioned tests were done.

Leukopenia is associated with decrease in plasma levels of interleukin (IL-7) and the frequency of its receptor were significantly decreased in preterm infants and in infection. Neonatal septicaemia is associated with leucopenia. The sensitivity of present study correlates with Makkar et al ${ }^{15}$, and Zaki et al $^{16}$ while negative predictive value also correlates with Zaki et al ${ }^{16}$ and Makkar et al. ${ }^{15}$ Specificity and positive predictive value correlates with Makkar et $\mathrm{al}^{15}$ and Buch et $\mathrm{al}^{9}$ respectively.

Absolute neutrophil count below $1800 /$ cubic $\mathrm{mm}$ is believed to be the best predictor of sepsis, while neutrophilia does not correlate well.

The present study showed 63.3\%, 74.2\%, 67.8\% and $70.2 \%$ of sensitivity, specificity, positive predictive value and negative predictive value respectively for absolute neutrophil count. The sensitivity of present study is comparable to Buch et $\mathrm{al}^{9}{ }^{9} \mathrm{Zaki}$ et $\mathrm{al}^{16} \mathrm{Basu}$ et $\mathrm{al}^{13}$ while negative predictive value correlates with Buch et al $^{9}$, Zaki et al. ${ }^{16}$ Specificity in the present study is comparable to Zaki et al. ${ }^{16}$ while Positive predictive value correlates with Hussein et al. ${ }^{17}$

The immature to total neutrophil count ratio has been one of the most extensively studied neutrophil index. Some authors have calculated the I:T ratio taking into account all the immature neutrophils including band form and obtained the ratio with mature to total neutrophil count, many authors have calculated by taking only the band neutrophil: Total neutrophil ratio wherein only the band neutrophils were considered in the numerator of the ratio. The present study correlates with Zaki et al, ${ }^{16}$ Nandy et $\mathrm{al}^{5}$ (2007). The present study also showed high sensitivity and low specificity compared to Nandy et al. ${ }^{5}$, Zaki et al. ${ }^{16}$ Sensitivity correlates with Zaki et al. ${ }^{16}$ Positive predictive value of present study is comparable to Nandy et $\mathrm{al}^{5}$ while negative predictive value is comparable to Zaki et al ${ }^{16}$ \& Nandy et al. ${ }^{5}$

Thrombocytopenia was frequently associated with sepsis and indicated poor prognosis. The present study observed $60 \%$ of sensitivity, $80 \%$ of specificity, $72 \%$ and $70 \%$ of positive and negative predictive value respectively. Sensitivity and negative predictive value correlates with Rekha Sriram. ${ }^{6}$ Specificity is comparable to Buch et al ${ }^{9}$ while positive predictive value correlates with Makkar et al. ${ }^{15} \mathrm{We}$ found in our study that thrombocytopenia is a good predictor of neonatal septicaemia as it had high sensitivity, specificity, positive and negative predictive value.

C-reactive protein, an acute phase protein in inflammation has been evaluated by many workers in this regard. But lack of specificity was the main disadvantage. CRP is considered positive if its value is $>1 \mathrm{mg} / \mathrm{dL}$. The present study observed high sensitivity, low specificity, high negative predictive value and low positive predictive value that is $88 \%, 68.5 \%, \quad 70.6 \%, 87.2 \%$ respectively. Sensitivity correlates with Zaki et al,16 Nandy et $\mathrm{al}^{4}$ while negative predictive value correlates with Zaki et al,16 Basu et al.13 Specificity is comparable to Buch et al, ${ }^{9}$ Sriram et al ${ }^{6}$ while positive predictive value correlates with Buch et al. ${ }^{9}$

The micro-ESR is a popular constituent of the screening tests undertaken to detect neonatal sepsis and has for long been recognised as a valid investigative tool. ESR, a nonspecific indicator of tissue damage is known to increase in infective states. The micro-ESR is generally normal or mildly elevated in non-infectious conditions, such as respiratory distress syndrome, aspiration pneumonitis and asphyxia, as well as in superficial infections.

Normal value of micro-ESR is: any value $>$ age (days) +3 up to maximum of $15 \mathrm{~mm} / \mathrm{hr}$.

In the present study, micro-ESR was least sensitive but highly specific and has low positive predictive value and high negative predictive value. The present study showed $26.6 \%$ sensitivity, $85.5 \%$ specificity, $61.5 \%$ positive predictive value and $57 \%$ negative predictive value. Sensitivity correlates with Hussein et al,17 Nandy et al ${ }^{5}$ while negative predictive value correlates with Buch et al. ${ }^{9}$ Specificity is comparable to Hussein et $\mathrm{al}^{17}$ Nandy et $\mathrm{al},{ }^{5}$ Basu et al ${ }^{13}$ while positive predictive value correlates with Basu et al. ${ }^{13}$

Many authors observed varying results for this test. These variations are due to the fact that at least four hours are required for haematological response to develop after the onset of infection, and blood samples collected and analysed before this will yield normal results. High micro-ESR is a specific test, but it has very low sensitivity. The value is spuriously high in neonates with haemolysis and low in babies with disseminated consumptive coagulopathy. ${ }^{6}$

\begin{tabular}{|c|c|c|c|c|}
\hline $\begin{array}{c}\text { Authors } \\
\text { (year) }\end{array}$ & Sensitivity & Specificity & $\begin{array}{c}\text { Positive } \\
\text { Predictive } \\
\text { Value }\end{array}$ & $\begin{array}{c}\text { Negative } \\
\text { Predictive } \\
\text { Value }\end{array}$ \\
\hline $\begin{array}{c}\text { Hussein } \\
\text { et al }{ }^{17} \\
(2007)\end{array}$ & 26.5 & 81 & 32.1 & 76.4 \\
\hline $\begin{array}{c}\text { Nandy et } \\
\text { al}^{5}(2007)\end{array}$ & $18 \%$ & $93.9 \%$ & $86.6 \%$ & $34.5 \%$ \\
\hline $\begin{array}{c}\text { Buch et } \\
\text { al}^{9}(2011)\end{array}$ & $63.08 \%$ & $72.73 \%$ & $73.21 \%$ & $62.5 \%$ \\
\hline $\begin{array}{c}\text { Present } \\
\text { study }\end{array}$ & 26.6 & 85.5 & 61.5 & 57 \\
\hline
\end{tabular}

Table 7. Showing Comparison of Diagnostic Test for Micro-ESR in the Present study with Other studies

Wilson et $\mathrm{al}^{7}$ stated that the organisms causing neonatal sepsis differ from place to place, country to country, and from nursery to nursery. In the same nursery, they vary from season to season. Low culture positivity may be because of 
intrapartum administration of antibiotics to mothers which can affect the blood culture reports in neonates.

Hussein ${ }^{17}$ (2007), Sriram ${ }^{6}$ reported low culture positivity $42 \%$ and $50.4 \%$ respectively. Khair et al ${ }^{18}$ (2010), and Shirazi et $\mathrm{al}^{12}$ (2010) reported low culture positivity $12 \%, 28.8 \%$, $35 \%$ respectively whereas Nandy et al ${ }^{5}$ (2007), Buch et $\mathrm{al}^{9}$ (2011) reported comparatively high culture activity $72 \%$ and $54.17 \%$ respectively.

Microbiological culture test was performed in all 130 cases of neonatal sepsis. $46.1 \%$ of cases were culture positive. Gram-negative organisms were the most common isolate in our study, Klebsiella being the most common. Some other authors reported Staphylococci as commonest isolate in their study. Present study was comparable to Buch et al $^{9}$ (2011) and Rekha Sriram ${ }^{6}$ (2011) studies.

\section{CONCLUSIONS}

- Neonatal sepsis was more common in preterm and low birth weight babies.

- There was male predominance in neonatal sepsis.

- Early onset sepsis (early neonatal period) was more common than late onset sepsis.

- Gram-negative infection was more common, Klebsiella was the most common organism isolated.

- C-reactive protein had highest sensitivity among all. Micro-ESR had highest specificity.

- Haematological profile test is cheap and easy to perform, less time consuming and can be employed for routine screening of all clinically suspected cases of neonatal septicaemia in developing countries where cost is a major limiting factor.

- All the tests employed using haematological profile can be performed easily at the primary health care centre level and results can be obtained in an hour.

\section{REFERENCES}

[1] Aggarwal R, Sarkar N, Deorari AK, et al. Sepsis in the newborn. Indian J Pediatr 2001;68(12):1143-7.

[2] Narang A. Epidemiology of neonatal sepsis. In: Dutta S, Narang A, (eds). Neonatal sepsis. Chandigarh: Pakeeza printers; 2003:1-7.

[3] Namdeo UK, Singh HP, Rajput VJ, et al. Hematological indices for early diagnosis of neonatal septicemia. Indian Pediatr 1985;22(4):287-92.

[4] Buch AC, Srivastava V, Kumar H, et al. Evaluation of haematological profile in early diagnosis of clinically suspected cases of neonatal sepsis. Int J Basic and Applied Sciences 2011;1(1):1-6.
[5] Gupta S, Aggarwal KC, Bhakoo ON. Evaluation of buffy coat examination in septicemia during infancy. Indian Pediatr 1987;24(1):49-51.

[6] Nandy M, Dutta S, Ganguly S, et al. Changing spectrum of neonatal septicemia. The Child and New Born 2007;11(1):3-6.

[7] Khair KB, Rahman MA, Sultana $T$, et al. Role of hematologic scoring system in early diagnosis of neonatal septicemia. BSMMU J 2010;3(2):62-7.

[8] Wilson HD, Eichenwald HF. Sepsis neonatorum. Pediatr Clin North Am 1974;21(3):571-82.

[9] Hammerschlag MR, Klein JO, Herschel M, et al. Patterns of use of antibiotics in two newborn nurseries. N Engl J Med 1977;296(22):1268-9.

[10] Swarnakar K, Swarnakar M. A study of early onset neonatal sepsis with special reference to sepsis screening parameters in a tertiary care centre of rural India. The Internet J Inf Dis 2012;10(1).

[11] Haider S, Riaz S, Rida T. Role of haematological profile in early diagnosis of neonatal sepsis. Ann Pak Inst Med Sci 2010;6(3):152-6.

[12] De A, Saraswati K, Gogate A, et al. C-reactive protein and buffy coat smear in early diagnosis of childhood septicemia. Indian J Pathol Microbiol 1998;41(1):23-6.

[13] Varsha, Rusia U, Sikka M, et al. Validity of haematological parameters in identification of early and late onset neonatal infection. Indian J Pathol Microbiol 2003;46(4):565-8.

[14] Basu R, Bandyopadhyay S. Study on correlation between sepsis screening and blood culture in neonatal sepsis. IOSR J Dent Med Sci 2014;13(5):52-6.

[15] Harmoinen A, Hällström O, Grönroos P. Rapid quantitative determining of C-reactive protein using laser-nephelometer. Scand J Clin Lab Invest 1980;40(3):293-5.

[16] Makkar M, Gupta C, Pathak R, et al. Performance evaluation of haematological scoring system in early diagnosis of neonatal sepsis. J Clin Neonatol 2013;2(1):25-9.

[17] Zaki Mel-S, el-Sayed H. Evaluation of microbiologic and hematologic parameters and E-selectin as early predictors for outcome of neonatal sepsis. Arch Pathol Lab Med 2009;133(8):1291-6.

[18] Sriram R. Correlation of blood culture results with the sepsis score and the sepsis screen in the diagnosis of neonatal septicemia. Int J Biol Med Res 2011;2(1):360-8. 